\title{
Pattern-onset visual evoked potentials in suspected multiple sclerosis
}

\author{
MICHAEL J AMINOFF AND ALFRED L OCHS \\ From the Department of Neurology, University of California School of Medicine, San Francisco, \\ California, and the Medical College of Virginia, Richmond, Virginia, USA
}

S U M MARY Visual evoked potentials (VEPs) were obtained by monocular stimulation using a checkerboard pattern-reversal and pattern-onset technique. In 11 normal subjects, pattern-onset VEPs were generally larger, better defined, and less ambiguous than those elicited by patternreversal, because of the biphasic waveform characteristically obtained with pattern-onset stimulation. In 68 of 105 patients with possible multiple sclerosis, VEPs were normal in latency by both methods, and in nine adequate comparison was not possible. The incidence of normal VEPs to pattern-reversal was similar to that found in several other studies of patients with possible multiple sclerosis. Among the remaining 28 patients in whom VEP abnormalities were found, an increased latency was detected in $75 \%$ with the pattern-reversal technique, and in $96 \%$ by pattern-onset. In these patients, VEP abnormalities were obtained by monocular stimulation of each of 46 eyes, and among these the pattern-reversal technique yielded abnormalities in $59 \%$ and the pattern-onset method in $98 \%$. These results indicate that VEPs elicited by pattern-onset are useful in investigating patients with suspected multiple sclerosis, and the diagnostic yield may be greater than with conventional pattern-reversal techniques.

In recent years the usefulness of the averaged visual evoked potential (VEP) in the diagnosis of multiple sclerosis and other disorders affecting the anterior visual pathway has been reported. In clinical practice, VEPs are generally elicited either by a flash of light or by the alternating reversal of a checkerboard pattern, ${ }^{1-4}$ the responses being averaged by means of a small computer. The VEPs elicited by pattern-reversal are more consistent and more dependably identified than those elicited by flash, ${ }^{2}$ perhaps because the pattern stimulates the lateral inhibitory networks of the visual system. ${ }^{5}$ Despite this advance, patternreversal VEPs are sometimes obscured by unrelated cortical potentials and can be difficult to interpret accurately in clinical practice because of uncertainty as to which wave constitutes the major positive peak, the component of particular interest. Moreover, it is still not clear whether this method of eliciting VEPs is the most sensitive one for detecting pathological involvement of the optic

Address for reprint requests: Dr MJ Aminoff, Department of Neurology, School of Medicine, University of California, San Francisco, California 94143, USA.

Accepted 23 March 1981 nerve. Indeed, Halliday ${ }^{6}$ has recently stressed the need for further studies to determine the optimal method of stimulation for clinical purposes.

A second method of pattern stimulation is to flash-illuminate the pattern, but the waveform of the response elicited in this way is similar to that elicited by unpatterned flash. VEPs may also be obtained by transiently interrupting a uniform grey field with a pattern. This presentation elicits separate pattern-onset and offset responses whose characteristics have been studied in several laboratories. $^{7-10}$ We have compared the VEP obtained with a standard checkerboard patternreversal stimulus with that obtained by patternonset of the same checkerboard in a group of normal subjects in order to determine whether the latter was more easily interpreted in practice. In order to evaluate the clinical relevance of the VEPs obtained by these two means, we have also compared the findings in a group of 105 patients with possible multiple sclerosis who were referred for electrophysiological evaluation to detect in. volvement of the anterior visual pathway. In describing our findings, we have used the nomenclature and units of measurement proposed in 
the 1974 Brussels Symposium on Cerebral Evoked Potentials. ${ }^{11}$ A preliminary account of our findings was presented at the 1980 meeting of the American EEG Society. ${ }^{12}$

\section{Methods}

Stimuli were projected from two $35 \mathrm{~mm}$ slides onto the back of a translucent projection screen after the method of Halliday and Michael. ${ }^{13}$ Each projector was fitted with an Ultrablitz electronic shutter to control stimulus presentation. These shutters opened completely in $3 \mathrm{~ms}$ and closed completely in $4.3 \mathrm{~ms}$ Control experiments showed that shutter clicks themselves did not produce an evoked response under the conditions detailed below. Both projectors were mounted side-by-side, and the optical distortion caused by their displacement from the perpendicular axis of the screen was compensated for by photographing the slides from an angle equal to that of the projector displacement. A square array of checkerboards subtending 10 degrees of visual angle, horizontally and vertically, and having a half-period of $50 \mathrm{~min}$ per check, was used as the stimulus for pattern-reversal and pattern-onset. Luminance of the bright squares was $415 \mathrm{ft}-\mathrm{L}\left(1425 \mathrm{Cd} / \mathrm{m}^{2}\right)$, of the black was $60 \mathrm{ft}-\mathrm{L}\left(205 \mathrm{Cd} / \mathrm{m}^{2}\right)$, and of the near background was $17 \mathrm{ft}-\mathrm{L}\left(60 \mathrm{Cd} / \mathrm{m}^{2}\right)$. The contrast between the black and bright areas, defined as [( $\max -$ $\min ) /(\max +\min )] \times 100$, was therefore $75 \%$.

In the standard pattern-reversal presentation, two checkerboard slides were caused to alternate by the stimulus trigger so that the black and white squares would interchange. In the pattern-onset presentation, the subject viewed a grey field until the stimulus was triggered, when the field was replaced transiently by the checkerboard for only the time necessary to obtain a VEP $(240 \mathrm{~ms})$. With presentation of the pattern stimulus for this duration, the pattern-onset VEP was not confounded with the offset response. ${ }^{14}$ The grey slide was made by superimposing a Kodak $0.3 \mathrm{D}$ neutral density Wratten filter on a piece of blank film, providing a luminance equal to the average luminance of the patterns. A fixation point was provided in the centre of the grey field. The order in which the recordings were made was randomised. Stimuli were triggered at irregular intervals, ranging from 1.0 to $3.5 \mathrm{~s}$, to avoid spurious reinforcement of late evoked potentials on the next stimulus presentation. The VEP was obtained from standard occipital $0_{1}$ and $0_{2}$ scalp electrode placement $(10-20$ International System), referenced to the vertex $\left(C_{z}\right)$. The low and high frequency filters of the EEG amplifiers were set at 0.05 and $100 \mathrm{~Hz}$. A $60 \mathrm{~Hz}$ notch filter $(\mathrm{Q}=3)$ was also used after pilot studies showed an improved signal-to-noise ratio without any effect on the timing or waveform of the evoked potential. 100 stimulus responses were averaged with a CAT Mnemotron computer, which was triggered $25 \mathrm{~ms}$ before the stimulus to establish a baseline. Calibration was performed before testing each subject by averaging a $10 \mu \mathrm{V}, 100 \mathrm{~ms}$ pulse. The recording arangements were such that occipital surface-positive potentials caused a downward deflection of the trace.

Eleven normal subjects (six male, five female), aged between 27 and 41 years, were examined fundoscopically for evidence of asymptomatic optic nerve involvement but no abnormalities were found. Each subject was tested monocularly with both the pattern-reversal and pattern-onset presentation, while in a supine position with the stimulus $1 \mathrm{~m}$ away. In addition, 105 patients ( 46 male, 59 female), ranging in age between 19 and 65 years, who were considered to have possible multiple sclerosis according to previously-published diagnostic criteria, ${ }^{15}$ and who were referred to this laboratory for electrophysiological evaluation to detect subclinical optic nerve involvement, were also tested by both methods. Subjects and patients were tested by both methods in random order at the same session.

\section{Results}

\section{Normal subjects}

The waveform and time-course of the potential usually evoked by the pattern-reversal stimulus was similar in all important aspects to that reported by other workers. ${ }^{416}$ This consisted of an occasional first positive wave, a low amplitude negative wave, and then a large (principal) positive wave occurring at about $100 \mathrm{~ms}$ after the stimulus. However, this waveform varied between subjects so that it sometimes became difficult to identify the principal positive wave with certainty. The form of the potential evoked by the checkerboard pattern-onset stimulus differed from that evoked by pattern-reversal. The prototype response was a large biphasic curve, with a principal positive peak at about $80 \mathrm{~ms}$, followed by a principal negative peak at about $110 \mathrm{~ms}$. Similar patternonset VEP waveforms are reported by Estevez and Spekreijse. ${ }^{10}$ As with the pattern-reversal stimulus, the checkerboard pattern-onset presentation also gave rise to idiosyncratic variations in the response. When viewing pattern-onset stimuli, subjects reported complete disappearance of the pattern after-image before presentation of the next stimulus pattern. Representative comparison of waveforms elicited from two subjects by each of these techniques are shown in the figure. The potentials evoked by pattern-onset in each of the 11 subjects were compared with those elicited by the checkerboard pattern-reversal technique.

In analysing the data, latency was measured to the peak of the principal positive wave elicited by pattern-reversal, and to the peaks of the principal positive and negative waves evoked by pattern- 


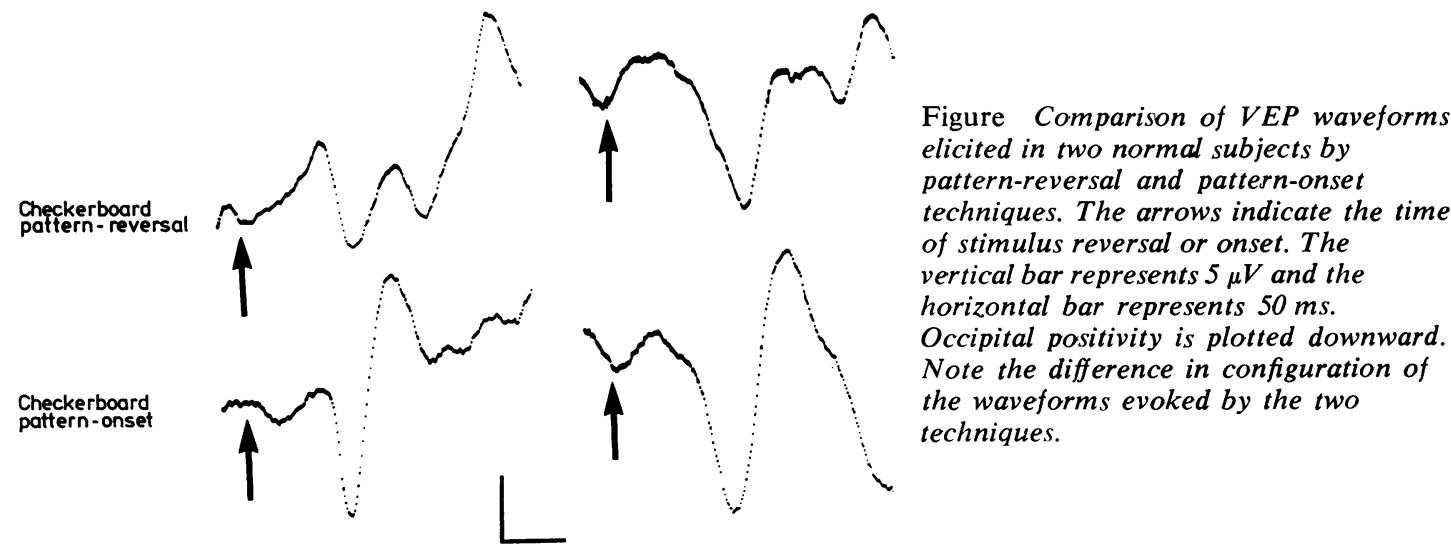

onset stimulation. We have chosen to measure the magnitude of an individual peak from the immediately preceding wave of opposite sign, as have other workers. ${ }^{24}$ The mean amplitude of the principal waves comprising the VEP elicited by the checkerboard pattern-reversal and patternonset techniques are shown in the table for comparative purposes. The average amplitude of the principal positive component of the VEP elicited by pattern-reversal was somewhat larger than that elicited by pattern-onset, but this difference was not statistically significant $(p=0.0514)$. The measured amplitude of the principal negative wave evoked by the pattern-onset stimulus was large, however, because of the usual biphasic waveform of the responses. Because of this characteristic biphasic waveform, we found that the patternonset response was generally the easier to read since there was little ambiguity as to which was the peak of interest. Responses were judged to be more easily recognisable with the checkerboard pattern-onset stimulus than the pattern-reversal stimulus in six of the 11 subjects, recognisable with equal facility in two subjects, and less easily discerned in three subjects. Although assessments were made independently, there was complete agreement between the two authors.

The latency of the principal positive peak elicited by pattern-onset presentation of the checkerboard stimulus was significantly shorter than that elicited by pattern-reversal of the same checkerboard $(p=0.0001)$ as shown in the table.

Patients with possible multiple sclerosis

In 68 of the 105 patients with possible multiple sclerosis, pattern-reversal and pattern-onset VEPs obtained by monocular stimulation of each eye were of normal configuration and had a latency within 2.5 standard deviations of the mean as determined in normal subjects (table). In nine other patients, the record obtained did not permit adequate comparisons to be made; in five of these patients the responses obtained from each eye were uninterpretable irrespective of the stimulation technique employed, because they differed so markedly from the normal configuration or were obscured by artifact. In a further two patients, responses to pattern-reversal were clearly normal in configuration and latency, while those to pattern-onset were technically unsatisfactory and uninterpretable; in two other patients, the converse was true. These nine patients are omitted

Table Comparison of VEPs elicited in 11 normal subjecis by pattern-reversal and pattern-onset techniques. The Fisher paired-t test was used to measure significance of differences between the two techniques

\begin{tabular}{|c|c|c|c|c|c|}
\hline & \multicolumn{2}{|c|}{ Pattern-reversal } & \multicolumn{2}{|c|}{ Pattern-onset } & \multirow{2}{*}{$\begin{array}{l}\text { Significance } \\
\text { of difference }\end{array}$} \\
\hline & Mean & $\begin{array}{l}\text { Standard } \\
\text { deviation }\end{array}$ & Mean & $\begin{array}{l}\text { Standard } \\
\text { deviation }\end{array}$ & \\
\hline $\begin{array}{l}\text { Latency of principal positive peak }(\mathrm{ms}) \\
\text { Amplitude of principal positive peak }(\mu \mathrm{V}) \\
\text { Latency of principal negative peak }(\mathrm{ms}) \\
\text { Amplitude of principal negative peak }(\mu \mathrm{V})\end{array}$ & $\begin{array}{r}97 \cdot 5 \\
8 \cdot 2\end{array}$ & $\begin{array}{l}7 \cdot 5 \\
4 \cdot 5\end{array}$ & $\begin{array}{r}78 \cdot 5 \\
5 \cdot 1 \\
107 \cdot 0 \\
10 \cdot 1\end{array}$ & $\begin{array}{l}4 \cdot 7 \\
3 \cdot 5 \\
9 \cdot 0 \\
4 \cdot 0\end{array}$ & $\begin{array}{l}0.0001 \\
0.0514\end{array}$ \\
\hline
\end{tabular}


from further consideration in this study. In the remaining 28 patients, monocularly elicited VEPs obtained by either or both stimulation techniques were prolonged in latency to beyond 2.5 standard deviations above the mean value for normal subjects in at least one eye. In 20 of these patients a response of prolonged latency was found by both techniques, and among these patients the abnormality was bilateral in six instances. In the other 14 of these 20 patients delayed VEPs to patternreversal and pattern-onset were found unilaterally, and in five of these patients the responses obtained by stimulation of the other eye were of prolonged latency when elicited by pattern-onset but were normal with pattern-reversal. In seven additional patients, pattern-onset VEPs from both eyes were delayed, but the corresponding pattern-reversal VEPs were normal in each eye. In the remaining one patient a VEP of prolonged latency was found in response to pattern-reversal stimulation when the responses to pattern-onset were normal, and in this instance the finding was unilateral, the responses obtained with stimulation of the other eye by either method being entirely normal.

The incidence of abnormalities among the 96 patients with possible multiple sclerosis considered here was $22 \%$ with the conventional patternreversal technique for eliciting VEPs, and $28 \%$ with the pattern-onset method. Among the total of 192 eyes (96 patients) tested, the incidence of abnormalities with the pattern-onset technique was $23 \%$, and by pattern-reversal was $14 \%$.

Among the 28 patients in whom VEP abnormalities were found, abnormalities were detected with the pattern-reversal technique in $75 \%$, and detected by pattern-onset in $96 \%$. However, the presentation of our findings in this way does not fully demonstrate the higher diagnostic yield with the pattern-onset method, because some patients had bilateral VEP abnormalities which were not indicated by the pattern-reversal technique. If the responses obtained by stimulation of each eye among the 28 patients with VEP abnormalities are considered separately, abnormally delayed responses to both pattern-onset and patternreversal stimulation were found in 26 , to patternonset alone in 19, and to pattern-reversal alone in one, while the responses were normal in the remaining 10 of the 56 eyes tested. That is, VEP abnormalities were obtained by monocular stimulation of each of 46 eyes, and among these the pattern-onset technique yielded abnormalities in $98 \%$ and the pattern-reversal method in $59 \%$.

When VEPs of prolonged latency were found in response to pattern-onset stimulation, generally both the principal positive and principal negative waves were delayed. In only one instance was there a dissociation between these two peaks in this regard. The patient had a prolonged patternreversal VEP with monocular stimulation of the right eye, and with pattern-onset stimulation the principal positive wave was similarly prolonged in latency while the latency of the subsequent principal negative wave was just within 2.5 standard deviations of the mean in normal subjects

\section{Discussion}

The utility of evoked potential techniques in helping to establish a diagnosis of multiple sclerosis is beyond question. In patients with symptoms or signs of only a single neurological lesion, evidence for a multiplicity of lesions may depend on the detection by electrophysiological means of subclinical involvement at other sites in the nervous system, and such findings may obviate the need for time-consuming, expensive and possibly hazardous further investigations. The incidence of VEP abnormalities is lower in patients in the diagnostic category of possible multiple sclerosis ${ }^{15}$ than in patients with definite multiple sclerosis, tending to lie between 20 and $30 \%$ in the former, and between 75 and $97 \%$ in the latter group. ${ }^{17}$ This is unfortunate since it is for patients with possible multiple sclerosis that there is a need to establish the diagnosis with greater certainty.

The relatively low incidence of VEP abnormalities in patients with possible multiple sclerosis may be due to several factors. First, the actual incidence of multiple sclerosis among such patients may be fairly small. Second, the incidence of involvement of the anterior visual pathways is probably considerably less than at a more advanced stage of the disease. With these points in mind, it would be of considerable interest to follow a group of patients in the diagnostic category of possible multiple sclerosis, to determine the proportion that actually went on to develop the disease, and to correlate this with the VEP findings obtained when the diagnosis was uncertain. The third factor that may account for the relatively low incidence of VEP abnormalities in patients with possible multiple sclerosis is related to the sensitivity of the technique used; the method may not be sufficiently sensitive to detect very early involvement of the anterior visual pathways. In this latter regard, refinement in methodology may improve the yield, but necessitates comparison of the results of different techniques in the same patients. ${ }^{18} 19$ 
Nilsson, ${ }^{19}$ in a somewhat similar study to ours, compared the diagnostic yield of VEPs obtained by checkerboard pattern-reversal stimulation with those obtained by a display of light-emitting diodes (LEDs) generating a reversal of a pattern of illuminated red circular areas. In patients with possible multiple sclerosis, abnormal responses were found in a higher proportion when the LED stimulus was used, but recordings were less noisy and more clearly defined with the conventional technique, thereby permitting more reliable latency determinations. Hennerici and colleagues ${ }^{18}$ have also reported a comparative study of different VEP techniques. They compared conventional checkerboard pattern stimulation with a technique in which a small bright rectangle was placed in the visual axis and served as a foveal stimulus. The incidence of delayed VEPs was significantly higher with the latter technique. Others ${ }^{20}$ have found, however, that foveal stimulation, at least with a small checkerboard pattern (each square subtending $15 \mathrm{~min}$ arc), was clinically unacceptable because of the marked variability in the response obtained in the same patients at different times.

We have compared the VEPs elicited by reversal and onset of a checkerboard pattern stimulus in normal subjects and patients with possible multiple sclerosis. Although smaller checks than those we used are known to elicit a response of larger amplitude, we did not include them in this study because they were reported by others $^{321}$ to be less effective in distinguishing a healthy optic nerve from one affected by retrobulbar neuritis or multiple sclerosis, although the work of Duwaer and Spekreijse ${ }^{22}$ did not seem to substantiate this view. A practical advantage of using large checks in clinical examinations is that patients often present without proper optical correction, and with large checks the adverse effect on the VEP of their uncorrected vision is minimised. ${ }^{23}$

There is no general agreement about the optimal montage for recording VEPs. We recorded from $\mathrm{O}_{1}$ and $\mathrm{O}_{2}$ with reference to $\mathrm{C}_{2}$, a commonly used arrangement. Some prefer to record from $\mathrm{O}_{z}$, or from just above this point, but in fact there is a broad area in the occipital region-encompassing both $0_{z}$ and $0_{1}$ and $0_{2}-$ from which a large principal positive wave can be recorded in response to Dattern-reversal stimulation. ${ }^{24}$ We chose $C_{z}$ as the reference point as this is generally preferred to linked mastoids, and because the waveform of interest is usually of the same latency but opposite polarity at this site compared to that recorded occipitally; a differential amplifier consequently produces in-phase summation, and thus a response of maximal amplitude. ${ }^{25}$ Other authors have, however, preferred a mid-frontal location for the reference electrode.

We compared the results obtained in a group of 105 patients with possible multiple sclerosis, when the VEP was elicited by a checkerboard stimulus presented in two different ways. In nine patients, however, the recordings obtained did not permit adequate comparison of the two methods and these cases were therefore omitted from further consideration. We found unilateral or bilateral VEP abnormalities in $28 \%$ of our remaining 96 patients by pattern-onset stimulation. With a conventional pattern-reversal technique, however, unilateral or bilateral abnormalities were found in only 21 patients $(22 \%)$ and this corresponds closely to the incidence of abnormalities in this diagnostic category reported by others with this same technique. ${ }^{17}$ Accordingly, there is no reason to believe that the yield with patternreversal stimulation in our series was artificially low. Furthermore, although the standard deviation of the mean latency of the first major positive component of the response to pattern-reversal in our normal subjects was larger than in some published series, it was similar to-or smaller than-that reported in others. ${ }^{17}$

In all of the patients in our series who had an abnormal VEP, subsequent clinical developments and the results of other investigative procedures have so far remained compatible with the clinical diagnosis of possible multiple sclerosis, but a longer follow-up period is necessary for a more definitive statement to be made in this regard. It is pertinent to note, however, that so far no "falsepositive" cases have come to light, that is, in none of our multiple sclerosis suspects with an abnormal VEP by one or both methods has any other structural pathology since come to be recognised.

We found in normal subjects that the VEPs elicited by pattern-onset of a checkerboard stimulus were generally unambiguous and better defined than the responses obtained by pattern-reversal stimulation, and the latency to peak of the major positive wave was shorter. In patients with possible multiple sclerosis we found an increased incidence of VEP abnormalities with pattern-onset of the checkboard stimulus rather than patternreversal. This higher yield could reflect the smaller standard deviation of mean latency obtained with pattern-onset stimulation in normal subjects, for reduced normal variability should facilitate the recognition of pathology. To test this possibility, 
we re-analysed the VEPs elicited by patternreversal among the 46 eyes shown to have abnormal delays by either form of stimulation. By applying the smaller standard deviation of patternonset to the pattern-reversal mean latency, only four additional monocular responses fell into the abnormal category, raising the yield with patternreversal stimulation from $59 \%$ to $67 \%$. This artificially increased yield is still considerably less than the $98 \%$ yield of pattern-onset stimulation, suggesting that reduced normal variability cannot be held to account for the increased yield with the pattern-onset technique. We therefore believe that pattern-onset stimulation is more sensitive than pattern-reversal to pathology of the visual pathway.

In an earlier study, ${ }^{26}$ we showed that the differences in waveform and latency of the responses evoked by pattern-reversal and pattern-onset stimulation are due to adaptation to the stimulus when the pattern-reversal technique is used. We therefore suggest that when the visual system is adapted to the pattern, the pattern itself becomes less effective in detecting pathology within the visual pathway.

We are grateful to Ms Mary Mantle and Ms Marcia Jensen for technical assistance, and Ms Lily Morita for secretarial help.

\section{References}

1 Halliday AM, McDonald WI, Mushin J. Visual evoked response in diagnosis of multiple sclerosis. Br Med J 1973a; 4:661-4.

2 Halliday AM, McDonald WI, Mushin J. Delayed pattern-evoked responses in optic neuritis in relation to visual acuity. Trans Ophthal Soc UK 1973b; 93:31 5-24.

3 Milner BA, Regan D, Heron JR. Differential diagnosis of multiple sclerosis by visual evoked potential recording. Brain 1974; 97:755-72.

4 Asselman P, Chadwick DW, Marsden CD. Visual evoked responses in the diagnosis and management of patients suspected of multiple sclerosis. Brain 1975; 98:261-82.

5 Padmos P, Haauman JJ, Spekreijse H. Visually evoked cortical potentials to patterned stimuli in monkey and man. Electroencephalogr Clin Neurophysiol 1973; 35:153-63.

6 Halliday AM. New developments in the clinical application of evoked potentials. Electroencephalogr Clin Neurophysiol 1978; Suppl 34:10521.

7 Jeffreys DA. Axford JG. Source locations of pattern-specific components of human visual evoked potentials. I. Component of striate cortical origin. Exp Brain Res 1972a; 16:1-21.
8 Jeffreys DA, Axford JG. Source locations of pattern-specific components of human visual evoked potentials. II. Component of extrastriate cortical origin. Exp Brain Res 1972b; 16:22-40.

9 Jeffreys D. The physiological significance of pattern visual evoked potentials. In: Desmedt JE, ed. Visual Evoked Potentials in Man: New Developments. Oxford: Clarendon Press 1977; 134-67.

10 Estevez O, Spekreijse H. Relationship between pattern appearance-disappearance and pattern reversal responses. Exp Brain Res 1974; 19:233-8.

11 Desmedt JE. Visual Evoked Potentials in Man: New Developments. Oxford: Clarendon Press, 1977.

12 Ochs AL, Aminoff MJ. Pattern-reversal and pattern-onset visual evoked potentials in normal subjects and patients with possible multiple sclerosis. (Abstract) Electroencephalogr Clin Neurophysiol 1981; 51:27P.

13 Halliday AM, Michael WF. Changes in patternevoked responses in man associated with the vertical and horizontal meridians of the visual field. J Physiol (Lond) 1970; 208:499-513.

14 Cobb WA, Ettlinger G, Morton HB. Cerebral potentials evoked in man by pattern reversal and their suppression in visual rivalry. $J$ Physiol (Lond) 1968; 195:33-4P.

15 McAlpine D, Lumsden CE, Acheson ED. Multiple Sclerosis. A Reappraisal. Edinburgh, Livingstone, 1972.

16 Halliday AM, McDonald WI, Mushin J. Delayed visual evoked response in optic neuritis. Lancet 1972; 1:982-5.

17 Sokol S. Visual evoked potentials. In: Aminoff MJ, ed. Electrodiagnosis in Clinical Neurology. New York, Churchill Livingstone 1980; 348-69.

18 Hennerici M, Wenzel D, Freund H-J. The comparison of small-size rectangle and checkerboard stimulation for the evaluation of delayed visual evoked responses in patients suspected of multiple sclerosis. Brain 1977; 100:119-36.

19 Nilsson BY. Visual evoked responses in multiple sclerosis: comparison of two methods for pattern reversal. J Neurol Neurosurg Psychiatry 1978; 41:499-504.

20 Meienberg O, Kutak L, Smolenski C, Ludin HP. Pattern reversal evoked cortical responses in normals. A study of different methods of stimulation and potential reproducibility. $J$ Neurol 1979; 222:81-93.

21 Wilson WB, Keyser RB. Comparison of the pattern and diffuse-light visual evoked responses in definite multiple sclerosis. Arch Neurol 1980; 37:30-4.

22 Duwaer AL, Spekreijse H. Latency of luminance and contrast evoked potentials in multiple sclerosis patients. Electroencephalogr Clin Neurophysiol 1978; 45:244-58.

23 Harter MR, White CT. Evoked cortical responses to checkerboard patterns: effect of check size as 
a function of visual acuity. Electroencephalogr Clin Neurophysiol 1970; 28:48-54.

24 Lehmann D, Skrandies W. Reference-free identification of components of checkerboard-evoked multichannel potential fields. Electroencephalogr Clin Neurophysiol 1980; 48:609-21.

25 Shahrokhi F, Chiappa KH, Young RR. Pattern shift visual evoked responses. Two hundred patients with optic neuritis and/or multiple sclerosis. Arch Neurol 1978; 35:65-71.

26 Ochs AL, Aminoff MJ. The effect of adaptation to the stimulating pattern on the latency and wave form of visual evoked potentials. Electroencephalogr Clin Neurophysiol 1980; 48:502-8. 